\title{
Singular Hopf Bifurcations in DAE Models of Power Systems
}

\author{
Wieslaw Marszalek ${ }^{1}$, Zdzislaw Trzaska ${ }^{2}$ \\ ${ }^{1}$ College of Engineering \& Information Sciences, DeVry University, North Brunswick, USA \\ ${ }^{2}$ Department of Management and Production Engineering, Warsaw University of Ecology and Management, \\ Warsaw, Poland \\ E-mail:wmarszalek@devry.edu,zdzislaw.trzaska@netlandia.pl \\ Received August 25, 2010; revised October 10, 2010; accepted October 15, 2010
}

\begin{abstract}
We investigate an important relationship that exists between the Hopf bifurcation in the singularly perturbed nonlinear power systems and the singularity induced bifurcations (SIBs) in the corresponding differenttial-algebraic equations (DAEs). In a generic case, the SIB phenomenon in a system of DAEs signals Hopf bifurcation in the singularly perturbed systems of ODEs. The analysis is based on the linear matrix pencil theory and polynomials with parameter dependent coefficients. A few numerical examples are included.
\end{abstract}

Keywords: Power Systems, Singularly Perturbed Systems, DAEs, Bifurcations, Matrix Pencils

\section{Introduction}

In an effort to better understand dynamical properties of power systems, their stability features and the impact of various parameters, the DAEs approach seems to be very important as was shown in a number of recent papers (see for example [1-10]).

Some of these papers deal with the existence of Hopf bifurcations in the singularly perturbed systems of nonlinear ODEs. DAEs are closely related to singularly perturbed ODEs, therefore it is natural to expect similar types of behavior. However, there are also obvious dif-ferences between the qualitative properties of DAEs and singularly perturbed ODEs. One can mention, for example, the SIB phenomenon, which is present in DAEs and not in ODEs. Also, singularities in DAEs add anotherlayer of difficulty in the bifurcation analysis, in particular in the Hopf bifurcation. Because of a local nature of these phenomena, it is feasible to consider the singular Hopf bifurcation and the SIBs by analyzing certain properties of linear matrix pencils and their characteristic polynomials [9,11-14].

The matrix pencil and characteristic polynomial approach used in the analysis of the singularly perturbed systems and DAEs models has one obvious drawback: the available results give sufficient conditions only and, as of today, no significant results are available for multi-parameter bifurcations of the underlying DAEs. The fun- damental SIB theorem for DAEs reported in [9] has been slightly improved in $[12,13,15]$ to weaken some of the sufficient conditions and to include the case of secondorder slow subsystems (typical in power systems). In [16] the sufficient conditions of the SIB phenomenon were given for quasi-linear DAEs and other interesting applications of DAEs have been reported in $[17,18]$.

In this paper we extend some of the results presented in [1]. In particular, we present further studies of the singularly perturbed power systems, their SIB, singular Hopf bifurcations and interactions between them. The topic of bifurcations in power systems (in particular the Hopf bifurcation) is one of the most important and interesting ones, and, at the same time quite difficult, as any realistic power grids have thousands of buses, generators and other devices. Even small failures of various devices of secondary importance may yield catastrophic consequences, power outages, or unstable systems' behavior $[7,8]$.

Section 2 provides a short introduction into the DAEs and singularly perturbed ODEs as models of power systems. Section 3 constitutes the main part of this paper. First, we discuss the SIBs and Hopf bifurcations in the context of singularities of DAEs. Next, several results related to these bifurcations in power systems are given and illustrated by several numerical examples. Section 4 provides a summary of the results and a few suggestions for possible extensions. The three part appendix is an 
important addition as it provides several properties of linear matrix pencils and polynomials with parameter dependent coefficients that are frequently used in our analysis in Section 3.

\section{DAEs as Models of Power Systems}

The DAE model of a power system is as follows $[1,2$, $19,20]$

$$
\begin{aligned}
& M_{i} \frac{d^{2} \alpha_{i}}{d t^{2}}+D_{i} \frac{d \alpha_{i}}{d t}+f_{i}=P_{m i}, i=1, \cdots, N_{G} \\
& f_{i}=P_{d i}, i=N_{G}+1, \cdots, N_{G}+N_{P V} \\
& f_{i}=P_{d i}, g_{i}=Q_{d i}, i=N_{G}+N_{P V}+1, \cdots, N_{G}+N_{P V}+N_{P Q}
\end{aligned}
$$

where $f_{i}$ and $g_{i}$ form the load flow equations and are defined as $(\mathrm{n} \equiv \mathrm{NG}+\mathrm{NPV}+\mathrm{NPQ})$

$$
\begin{aligned}
& f_{i}=\sum_{j=1}^{n}\left[V_{i} V_{j} B_{i j} \sin \left(\alpha_{i}-\alpha_{j}\right)+V_{i} V_{j} G_{i j} \cos \left(\alpha_{i}-\alpha_{j}\right)\right] \\
& i=1, \cdots, n \\
& g_{i}=\sum_{j=1}^{n}\left[V_{i} V_{j} G_{i j} \sin \left(\alpha_{i}-\alpha_{j}\right)-V_{i} V_{j} B_{i j} \cos \left(\alpha_{i}-\alpha_{j}\right)\right] \\
& i=N_{G}+N_{V}+1, \cdots, n
\end{aligned}
$$

with the following notation:

$M_{i}$ is the rotor inertia of the $i$ th generator,

$D_{i}$ is the damping coefficient of the $i$ th generator,

$V_{i}$ is the voltage magnitude of the $i$ th bus,

$\alpha_{i}$ is the angle of the $i$ th bus,

$B_{i j}, G_{i j}$ are the transfer susceptances and conductances,

$P_{m i}$ is the turbine mechanical power injection of the $i$ th generator, and bus.

$P_{d i}, Q_{d i}$ are the real and reactive power loads at the $i$ th

The above system can easily be written in the following DAE form

$$
M \frac{d^{2} x}{d t^{2}}+D \frac{d x}{d t}=f(x, y, \lambda), 0=g(x, y, \lambda)
$$

with $x \equiv\left[\alpha_{1}, \cdots, \alpha_{N G}\right]^{T}, y \equiv\left[\alpha_{N G+1}, \cdots, \alpha_{n}, V_{1}, \cdots, V_{n}\right]^{T}, \lambda$ is the vector of $k$ parameters, and the matrices $M, D$ are positive and semi-positive definite, respectively.

One of the generator buses is usually considered as a swing bus. This allows us to introduce a set of new variables (relative angles) and reduce the number of differential equations in the first subsystem in (3) by one [3].

The DAE model (3) and its bifurcations are closely related to the behavior of the singularly perturbed ODE model of the form

$$
M \frac{d^{2} x}{d t^{2}}+D \frac{d x}{d t}=f(x, y, \lambda), \varepsilon \frac{d y}{d t}=g(x, y, \lambda)
$$

when $\varepsilon \neq 0$. We shall examine the singular Hopf bifurcation of (4) for small $0<\varepsilon<<1$.

The singularly perturbed ODEs (4), when linearized, yield linear matrix pencils. Either first or second order matrix pencils can be used depending on an individual preference (see Appendices 1.1 and 1.2 for more details). A second order matrix pencil would normally be preferred for power systems as it may directly lead to double SIB points $[1,21]$.

\section{Bifurcations of Power Systems at Singularities}

\subsection{The Singularity Induced Bifurcation}

The SIB is a phenomenon attributed exclusively to DAEs. Since DAEs are used quite often as models of electrical power systems, it is important to study the changes of stability of those systems to better understand their dynamical properties. This may help to prevent future failures and catastrophic events such as, for example, massive power outages. It is also known that DAEs may exhibit other types of bifurcations that are widely found in nonlinear ODEs. One of such bifurcations is the wellknown Hopf bifurcation when a linearized system has a pair of complex conjugate eigenvalues on the imaginary axis with all other eigenvalues lying off the axis. The bifurcating parameter may cause the subcritical or supercritical Hopf bifurcations. The bifurcation is called subcritical if the periodic solutions are unstable and supercritical if the periodic solutions are stable. The Hopf bifurcation in power systems has been analyzed in [2,22].

The singularity of nonlinear semi-explicit parameter dependent DAEs $\dot{x}=f(x, y, \lambda), 0=g(x, y, \lambda)$ occurs when $\operatorname{det}\left(g_{y}\right)=0$ for some $\left(x^{*}, y^{*}, \lambda_{0}\right) \in R^{n+m+1}, x(t) \in R^{n}, y(t)$ $\in R^{m}, \lambda \in R, \dot{x}=d x / d t$ and $g_{y}=d g / d y$. The $\lambda$ is a slowly varying parameter. Equivalently, the linear matrix pencil

$$
\left(\begin{array}{ll}
I & 0 \\
0 & 0
\end{array}\right),\left(\begin{array}{ll}
A & B \\
C & D
\end{array}\right)
$$

where $A(\lambda)=f_{x}, B(\lambda)=f_{y}, C(\lambda)=g_{x}, D(\lambda)=g_{y}$, has index greater than 1 at $\left(x^{*}, y^{*}, \lambda_{0}\right)$ (see Appendices 1.1 and 1.2).

For the singularly perturbed ODEs we have $\dot{x}=f(x, y$, $\lambda), \varepsilon \dot{y}=g(x, y, \lambda)$ where $0<\varepsilon<<1$ is small and constant. A natural extension to second-order DAEs and singularly perturbed ODEs is to have $\ddot{x}=f(\dot{x}, x, y, \lambda), \varepsilon \dot{y}=g(x, y$, $\lambda)$.

It turns out that under the assumption of the SIB theorem (with an algebraically simple zero of $g_{y}\left(x^{*}, y^{*}, \lambda_{0}\right)$ [9]), the SIB phenomenon for semi-explicit parameter 
dependent DAEs may be equivalent to Hopf bifurcation of the singularly perturbed ODEs. The following example illustrates such a case.

Example 1: Consider the following singularly perturbed parameter dependent DAEs

$$
\dot{x}=\lambda-y, \varepsilon \dot{y}=x-\frac{1}{2} y^{2}
$$

Two solutions of (6) for $\lambda=0$ are shown in Figures 1-2. If $\varepsilon=0$, then the DAEs (6) undergoes the SIB as the parameter $\lambda \rightarrow 0$. The equilibrium locus is $\left(\lambda^{2} / 2, \lambda\right)$, i.e. $x=$ $\lambda^{2} / 2, y=\lambda$, and an eigenvalue $-1 / \lambda$ diverges through infinity as $\lambda \rightarrow \lambda_{0}=0$. The stability of the system is changed at $\lambda_{0}$. The point $\lambda_{0}$ is called a single SIB point (see [11]). The system is asymptotically stable for $\lambda>\lambda_{0}$ and unstable for $\lambda<\lambda_{0}$. This relatively simple case with scalar $f(x, y, \lambda)$ and $g(x, y, \lambda)$ is covered by Lemma 1 in [15] which, under the conditions $g_{y}\left(\lambda_{0}\right)=0, g_{y}^{\prime}\left(\lambda_{0}\right) \neq 0$ along the equilibrium locus, and $g_{x}\left(\lambda_{0}\right) f_{y}\left(\lambda_{0}\right)=-\omega^{2}<0$, predicts a pair of complex eigenvalues

$$
\alpha(\varepsilon, \lambda(\varepsilon)) \approx \pm \frac{i \omega}{\sqrt{\varepsilon}}\left(1-\frac{\varepsilon f_{x}^{2}\left(\lambda_{0}\right)}{\omega^{2}}\right)^{2}+O(1)
$$

as $\varepsilon \rightarrow 0$. The ${ }^{\prime}=d / d \lambda$ and $i=\sqrt{-1}$.

For (6) we have $f_{x}\left(\lambda_{0}\right)=0, g_{y}(\lambda)=-\lambda, g_{y}^{\prime}(\lambda)=-1$ and $\omega^{2}=1$. Thus, the eigenvalues (7) are: $\alpha(\varepsilon, \lambda(\varepsilon)) \sim \pm \omega \sqrt{ } \varepsilon+$

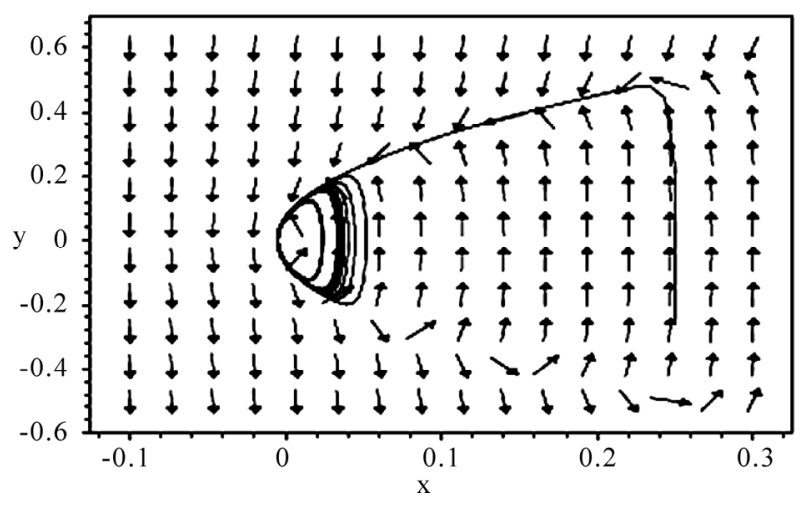

Figure 1. Trajectories for $\varepsilon=\mathbf{0 . 0 1}$.

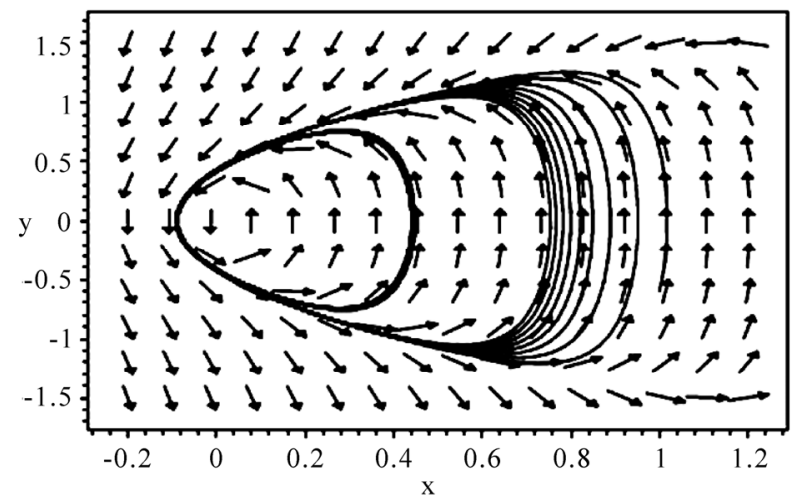

Figure 2. Trajectories for $\varepsilon=0.09$.
$O(1)$. The linearization of these DAEs along the equilibrium locus $\left(\lambda^{2} / 2, \lambda\right)$ yields the matrix pencil (see Appendix A)

$$
\left(\begin{array}{ll}
1 & 0 \\
0 & 0
\end{array}\right),\left(\begin{array}{cc}
0 & 1 \\
-1 & \lambda
\end{array}\right)
$$

which has index 1 for $\lambda \neq 0$ and index 2 for $\lambda=0$ (Appendix A). The $C B=-1, v=1$, and $C B v \notin \operatorname{Im}(D)$ at $\lambda=$ 0 . Therefore, at $\lambda=0$, the index jumps by one, the well established fact for DAEs undergoing the SIB. Also, the characteristic equation of the pencil (8) is (see Appendix 1.1 , case $k=1)-\lambda s+1=0$ with the $\operatorname{root} s=1 / \lambda$ diverging through $\pm \infty$ as $\lambda \rightarrow 0$.

Example 2: Now, suppose that $d^{2} x / d t^{2}+\gamma \mathrm{dx} / \mathrm{dt}=\lambda-y$, $\varepsilon d y / d t=x-1 / 2 y^{2}$, with $\gamma>0$. The eigenvalues are given by $-\gamma / 2 \pm \sqrt{(\gamma / 2)^{2}-1 / \lambda}$, and the system is asymptotically stable for $\lambda>0$ and unstable for $\lambda<0$. It can be checked that the linear system has index 1 if $\lambda \neq 0$, and the index jumps to 3 at $\lambda=0$. If $\lambda \neq 0$, then along the equilibrium locus $\left(\lambda^{2} / 2, \lambda, \lambda\right)$ we have (see Appendix 1.2): $\operatorname{det}(\mathrm{E}(0) \mathrm{s}-\mathrm{L}(\lambda))=s^{2} \lambda+s \gamma \lambda+1$, while at the SIB point $\lambda$ $=0$ we have $\operatorname{det}(E(0) s-L(0))=1$. This drop by 2 in the degree of $\operatorname{det}(E(0) s-L(\lambda))$ is equivalent to the index increase from 1 (at $\lambda \neq 0$ ) to 3 (at $\lambda=0$ ). If $\lambda<0$ one of the eigenvalues is unstable while the other remains stable. At $\lambda=0$ the eigenvalues diverge through $\pm \infty$. The point $\lambda=0$ is called the double SIB point, the concept introduced by Beardmore in [11].

For $\lambda \neq 0$ the Kronecker Normal Form of the pencil $(E(0), L(\lambda))$ is

$$
E(0)=\left(\begin{array}{lll}
1 & 0 & 0 \\
0 & 1 & 0 \\
0 & 0 & 0
\end{array}\right), L(\lambda)=\left(\begin{array}{ccc}
0 & 1 & 0 \\
-1 / \lambda & -\gamma & 0 \\
0 & 0 & 1
\end{array}\right)
$$

and for $\lambda=0$ the structure of the pencil changes to

$$
E=\left(\begin{array}{lll}
0 & 1 & 0 \\
0 & 0 & 1 \\
0 & 0 & 0
\end{array}\right), L(0)=\left(\begin{array}{lll}
1 & 0 & 0 \\
0 & 1 & 0 \\
0 & 0 & 1
\end{array}\right)
$$

\subsection{Properties of DAEs with SIB Phenomenon}

The main theorem indicating Hopf bifurcation in the singularly perturbed ODEs is due to Beardmore [15], as follows.

THEOREM: Suppose that the singularly perturbed ODEs $\dot{x}=f(x, y, \lambda), \varepsilon \dot{y}=g(x, y, \lambda)$ have a trivial equilibrium for $\lambda=\lambda_{0}$ and

1) $\operatorname{ker}\left(g_{y}\left(\lambda_{0}\right)\right)=\langle v\rangle$ with $v \notin \operatorname{Im}\left(g_{v}\left(\lambda_{0}\right)\right)$,

2) $g_{y}^{\prime}\left(\lambda_{0}\right)(v) \notin \operatorname{Im} g_{y}\left(\lambda_{0}\right)$, 
3) $\operatorname{det}\left[\begin{array}{ll}f_{x} & f_{y} \\ g_{x} & g_{y}\end{array}\right] \neq 0$

4) $\operatorname{ker}\left(g_{y}\left(\lambda_{0}\right)^{T}\right)=\langle u\rangle$ and

$\frac{u^{T} g_{x}\left(\lambda_{0}\right) f_{y}\left(\lambda_{0}\right) v}{u^{T} v}=-\omega^{2}<0$.

Then, there exists $\varepsilon_{1}>0$ such that $\forall \varepsilon_{1}>\varepsilon>0$ there is a $\lambda_{0}(\varepsilon)$ such that the singularly perturbed linearized ODEs have a pair of purely imaginary eigenvalues at $\lambda=\lambda_{0}(\varepsilon)$ and $\lambda_{0}(\varepsilon)$ is continuous in $\varepsilon$ at 0 with $\lambda_{0}(0)=\lambda_{0}$.

In the above, the ker, Im and det denote the kernel, image and determinant of a matrix, respectively, the $<>$ is a column vector and $T$ denotes the transposition. It is easy to check that the above sufficient conditions are satisfied for (6) with $v=u=1, g_{y}^{\prime}(0)=-1, f_{x} g_{y}-f_{y} g_{x}=1, g_{x}(0) f_{y}(0)$ $=-1$, yielding $\omega=1$.

LEMMA 1: Suppose that in (18) in Appendices 1.2 we have for $\varepsilon=0$ : $\operatorname{ind}(E(0), L(\lambda))=1$ for $\lambda \neq \lambda_{0}, \operatorname{det} L\left(\lambda_{0}\right) \neq 0$, $\operatorname{ker} D\left(\lambda_{0}\right) \equiv v \in R^{m}, D^{\prime}\left(\lambda_{0}\right) v \notin \operatorname{Im} D\left(\lambda_{0}\right)$ and $C\left(\lambda_{0}\right) B\left(\lambda_{0}\right) v \notin$ $\operatorname{ImD}\left(\lambda_{0}\right)$. Then,

$\operatorname{det}(E(0) s-L(\lambda))=a_{n-m}(\lambda) s^{n-m}+\cdots+a_{1}(\lambda) s+a_{0}(\lambda)$,

$$
\operatorname{det}\left(E(0) s-L\left(\lambda_{0}\right)\right)=s^{n-m-2}+\cdots+d_{1}\left(\lambda_{0}\right) s+d_{0}\left(\lambda_{0}\right),
$$

and the two eigenvalues of the matrix pencil $(E(0), L(\lambda))$ diverging through $\pm \infty$ are the zeros of the second-degree polynomial

$$
a(\lambda) s^{2}+b(\lambda) s+c(\lambda) \equiv \frac{a_{n-m}(\lambda) s^{n-m}+\cdots+a_{0}(\lambda)}{s^{n-m-2}+\cdots+d_{0}(\lambda)}
$$

with $a(\lambda)=a_{\mathrm{n}-\mathrm{m}}(\lambda), a\left(\lambda_{0}\right)=b\left(\lambda_{0}\right)=0$, and $c\left(\lambda_{0}\right) \neq 0$. The index of $\left(E(0), L\left(\lambda_{0}\right)\right)$ is 3 .

Lemma 1 is a consequence of the above theorem. In particular, from the Kronecker Normal Form of $(E(0)$, $L(\lambda))$ and the assumption that $\operatorname{ind}(E(0), L(\lambda))=1$ for $\lambda \neq \lambda_{0}$, we obtain (11). The other assumptions are needed to have the two eigenvalues divergent through $\pm \infty$ [11], and as a consequence, for $\lambda=\lambda_{0}$ we have (12).

Notice that in example $2 \operatorname{det}(E(0), L(\lambda))=s^{2} \lambda+s \gamma \lambda+1$ and $\operatorname{det}(E(0), L(0))=-1$, therefore $a(\lambda)=\lambda, b(\lambda)=\gamma \lambda, c(\lambda)$ $=1$ and the two diverging eigenvalues are the roots of $s^{2} \lambda$ $+s \gamma \lambda+1=0$. As a direct consequence of the above lemma we have the following result.

LEMMA 2: If the assumptions of lemma 1 are satisfied and if $\Gamma(\lambda) \equiv 0$ in (18) (see Appendix 1.2), then $b(\lambda) \equiv$ 0 and the two diverging eigenvalues are purely imaginary. If $c(\lambda) / a(\lambda)$ changes its sign from positive to negative at $\lambda$ $=\lambda_{0}$, then the system undergoes transition from a center to a saddle, or vice-versa, from a saddle to a center if $c(\lambda) / a(\lambda)$ changes sign from negative to positive.
The proof of the above lemma follows from the fact that the two diverging eigenvalues are $s_{1,2}=\sqrt{-c(\lambda) / a(\lambda)}$. No damping term exists in the system. A similar behavior in active RLC circuits has been reported in [23].

\subsection{An Illustrative Example}

The following example illustrates the above described features of a power system undergoing the singularity induced bifurcation.

Example 3: Consider the 4-bus, 3 generator power system shown in Figure 3. If we assume that the bifurcation parameter $\lambda$ defines the real and reactive powers at bus 4 with $P=P_{0}(1+\lambda), Q=Q_{0}(1+\lambda),\left(P_{0}=\right.$ const, $Q_{0}=$ const), then the DAE model (3) of this power system undergoes the SIB at $\lambda_{0}=0.537305$.

Assuming that the rotor inertia of all generators equals 1 , the line transfer conductances $G_{i j}$ are all zero, the damping generator coefficients to be $\gamma$, and considering bus 1 as a swing bus, the DAE system is as follows

$$
\begin{aligned}
& \frac{d^{2} \theta_{1}}{d t^{2}}+\gamma \frac{d \theta_{1}}{d t}=P_{m 2}-P_{m 1}-2 B_{21} V_{1} V_{2} \sin \left(\theta_{1}\right) \\
& -B_{13} V_{1} V_{3} \sin \left(\theta_{2}\right)+B_{24} V_{2} V_{4} \sin \left(\theta_{3}-\theta_{1}\right) \\
& \frac{d^{2} \theta_{2}}{d t^{2}}+\gamma \frac{d \theta_{2}}{d t}=P_{m 3}-P_{m 1}-2 B_{13} V_{1} V_{3} \sin \left(\theta_{2}\right) \\
& -B_{12} V_{1} V_{2} \sin \left(\theta_{1}\right)+B_{34} V_{3} V_{4} \sin \left(\theta_{3}-\theta_{2}\right) \\
& 0=B_{42} V_{2} V_{4} \sin \left(\theta_{3}-\theta_{1}\right)+B_{43} V_{3} V_{4} \sin \left(\theta_{3}-\theta_{2}\right)-P \\
& 0=B_{42} V_{4} V_{2} \sin \left(\theta_{3}-\theta_{1}\right)+B_{43} V_{3} V_{4} \sin \left(\theta_{3}-\theta_{2}\right)+B_{44} V_{4}^{2}+Q
\end{aligned}
$$

with the relative angles $\theta_{i} \equiv \alpha_{i+1}-\alpha_{1}$ for $i=1,2,3$.

Suppose that the parameters are: $B_{12}=B_{13}=1, B_{24}=B_{34}$ $=2, B_{44}=-4, B_{\mathrm{ij}}=B_{\mathrm{ji}}, P_{m 2}-P_{m 1}=1, P_{m 3}-P_{m 1}=2, P_{0}=1$, $Q_{0}=0.3, \gamma=1.5$ and $V_{i}=1$ for $i=1,2,3$. Then, the DAEs (14) have an equilibrium placed at the singularity for

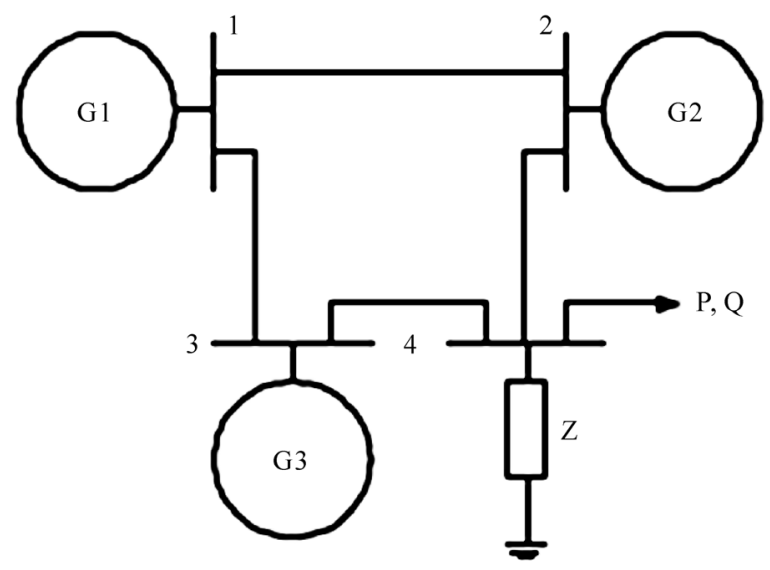

Figure 3. A simple 4-bus, 3-generator power system. 
$\left(\theta_{1}, \theta_{2}, \theta_{3}, V_{4}, \lambda\right)=(0.8496,2.3761,2.4940,0.6334,0.5373)$

For the singularly perturbed DAEs with $P=P_{0}(1+\lambda)$ and $Q=Q_{0}(1+\lambda)+\varepsilon d \theta_{4} / d t$ we get the same equilibrium. Solutions $\theta_{i}(t), i=1,2,3$, for $\varepsilon=0.002$ and various values of $\gamma$ are shown in Figure 4. Notice that $V_{4}(t)$ can be eliminated from (14) and the system reduces to two second-order differential equations and one first-order differential equation containing $\varepsilon d \theta_{4} / d t$. It turns out that the above equilibrium is unstable and at $\lambda=0.537305, \varepsilon=$

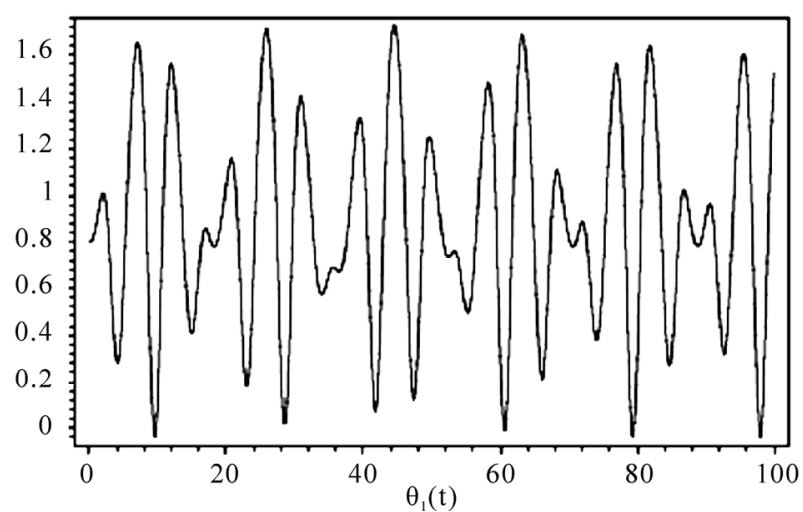

(a)

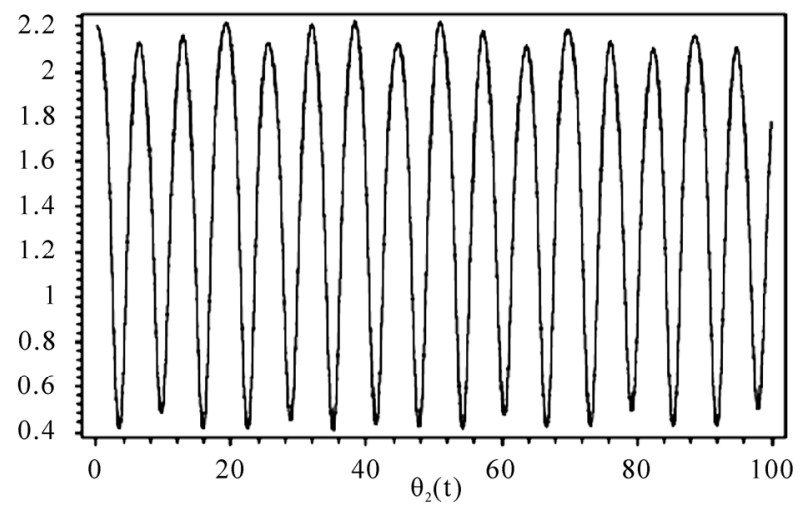

(c)

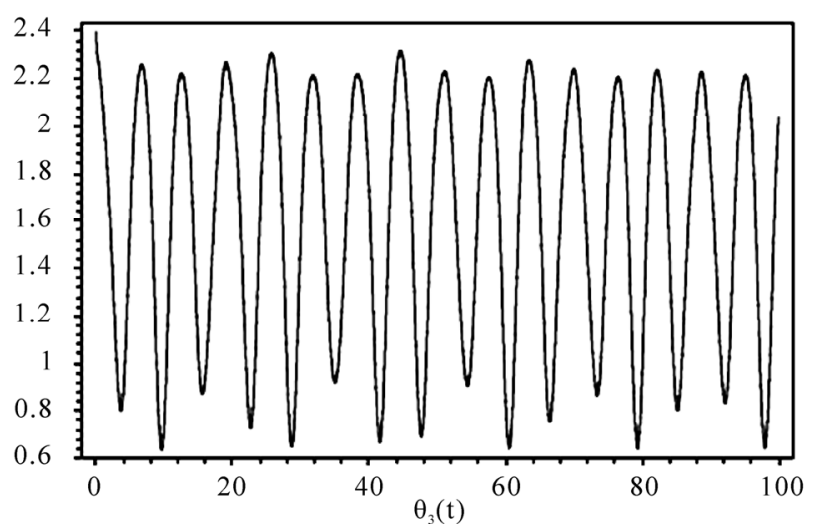

(e)
$0.002, \gamma=0$ the pencil (18) has the following eigenvalues $-5.6245 \pm 9.7933 i, \pm 0.1342 i, 11.2487$.

The solutions in Figure 4 escape the above equilibrium and oscillate around another equilibrium (not on the singularity)

$\left(\theta_{1}, \theta_{2}, \theta_{3}, V_{4}, \lambda\right)=(0.6812,1.0816,1.2770,1.1018,0.5373)$

with the eigenvalues $\pm 1.3238 i, \pm 1.5720 i,-4742.9260$ for $\gamma=0$ and $\varepsilon=0.002$.

For $\varepsilon=0$ and $\gamma \neq 0$ the characteristic equation of the

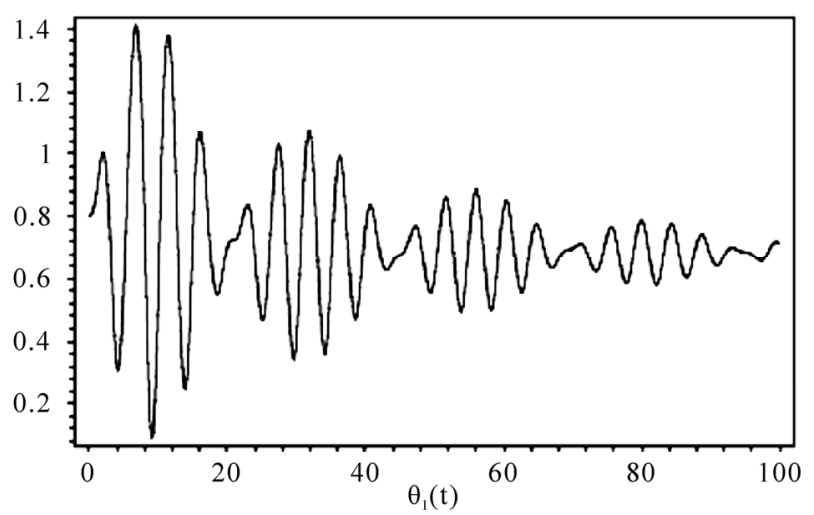

(b)

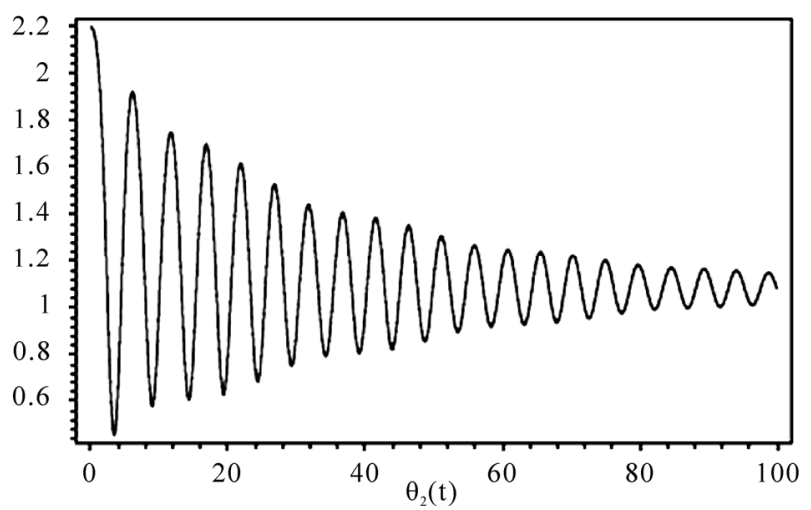

(d)

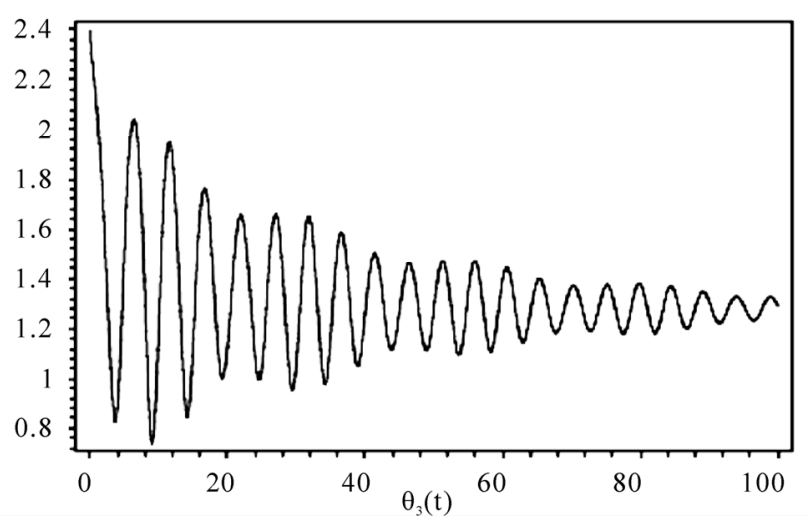

(f)

Figure 4. (a, c, e) The $\theta_{i}(t), i=1,2,3$, for $\gamma=0 ;(b, d, f)$ the $\theta_{i}(t), i=1,2,3$, for $\gamma=0.05$. 
linear matrix pencil (18) is (see (19)-(21) in Appendix 1.3): $a_{4}(\lambda) s^{4}+a_{3}(\lambda) s^{3}+a_{2}(\lambda) s^{2}+a_{1}(\lambda) s+a_{0}(\lambda)=0$ and for $\lambda=\lambda_{0}=0.537305$ the equation reduces to $2.869398 s^{2}+$ $4.304097 s+0.051705$.

Clearly, we have the case $k=2$ (see Appendix 1.3) and in (21) we have $c_{2}\left(\lambda_{0}\right)=c_{1}\left(\lambda_{0}\right)=0, c_{0}\left(\lambda_{0}\right)=2.869398$, $b_{1}\left(\lambda_{0}\right)=\gamma=1.5000$, and $b_{0}\left(\lambda_{0}\right)=0.018019$. For $\lambda \rightarrow \lambda_{0}$ the DAEs have two eigenvalues (divergent through infinity)

$$
s_{1,2}=-\frac{c_{1}(\lambda)}{2 c_{2}(\lambda)} \pm \sqrt{\frac{c_{1}^{2}(\lambda)}{4 c_{2}^{2}(\lambda)}-\frac{c_{0}(\lambda)}{c_{2}(\lambda)}}
$$

whose real parts are such that

$\lim _{\lambda \rightarrow \lambda_{0}}\left(-c_{1}(\lambda) /\left(2 c_{2}(\lambda)\right)\right)=-\gamma$ and the imaginary parts diverge to infinity as $\lambda \rightarrow \lambda_{0}$. The point $\lambda_{0}=$ 0.537305 is called a double SIB point and the linear matrix pencil has index 3 at $\lambda=\lambda_{0}$ and 1 if $\lambda \neq \lambda_{0}$.

One can also examine matrix $R(\lambda)$ in Appendix 1.4 to check that $\operatorname{det} R(\lambda)=0$ if the characteristic polynomial of the matrix pencil (18) admits a pair of purely imaginary roots. Figure 5 shows $\operatorname{det} R(\lambda)$ for system (14). Clearly, this determinant is 0 for $\lambda=0.537305$ because of the two complex eigenvalues $\pm 0.1342 i$ of the matrix pencil.

\section{Conclusions}

In the paper we have examined sufficient conditions under which the single and double SIB points in systems of DAEs are responsible for the Hopf bifurcation in singularly perturbed systems of ODEs. Both, the DAEs and singularly perturbed ODEs are widely used as dynamical models of power systems making their analysis very relevant in the studies of stability, reliability and sensitivity to various parameters. The analysis of power system models around their singularities is important from both the qualitative and numerical points of view. The qualitative analysis presented in this paper should be supplemented by a numerical one [17], as a typical power system

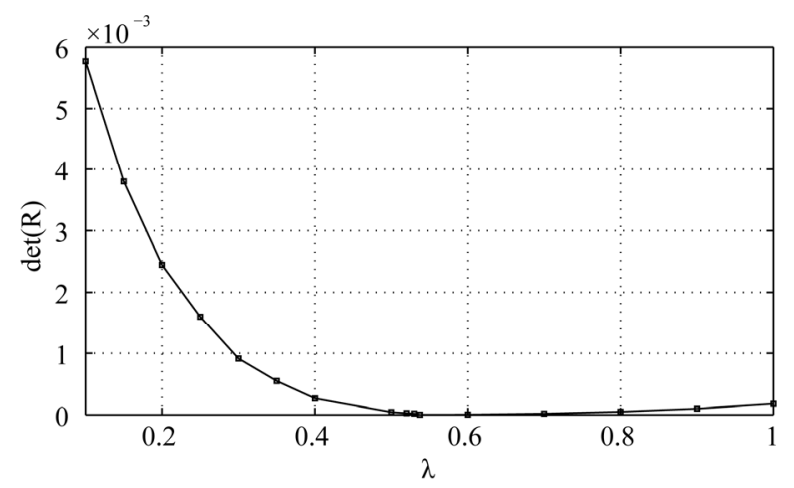

Figure 5. The det $R(\lambda)$ as a function of $\lambda$ (see Appendices 1.4). model includes hundreds or even thousands of equations [20].

It is also our opinion that the future work in the qualitative analysis of the SIB and singular Hopf phenomena via matrix pencils and parameter dependent polynomials should focus on the formulation of the necessary and sufficient conditions and the analysis of multi-parameter bifurcations at singularities. To the authors' knowledge no formulation of the necessary conditions of the SIB phenomenon exists at the present time.

\section{References}

[1] W. Marszalek and Z.W. Trzaska, "Singularity-Induced Bifurcations in Electrical Power Systems," IEEE Transactions on Power Systems, Vol. 20, No. 1, 2005, pp. 312320. doi:10.1109/TPWRS.2004.841244

[2] H. G. Kwatny, R. F. Fischl and C. O. Nwankpa, "Local Bifurcation in Power Systems: Theory, Computation, and Applications," Proceeding of IEEE, Vol. 83, No. 11, 1995, pp. 1456-1483. doi:10.1109/5.481630

[3] H. G. Kwatny, A. K. Pasrija and L. Y. Bahar, "Static Bifurcations in Electric Power Networks: Loss of Steadystate Stability and Voltage Collapse," IEEE Transactions on Circuits and Systems, Vol. CAS-33, No. 10, 1986, pp. 981-991.

[4] H. G. Kwatny and X.-M. Yu, "Energy Analysis of Load-Induced Flutter Instability in Classical Models of Electric Power Networks," IEEE Transactions on Circuits and Systems, Vol.36, No.12, 1989, pp. 1544-1557.

[5] S. Ayasun, C. O. Nwankpa and G. G. Kwatny, "Computation of Singular and Singularly Induced Bifurcation Points of Differential-Algebraic Power System Model," IEEE Transactions on Circuits and Systems I, Vol. 51, No. 8, 2004, pp. 15251538.

[6] D. J. Hill and I. M. Y. Mareels, "Stability Theory for Differential/Algebraic Systems with Application to Power System," IEEE Transactions on Circuits and Systems, Vol. CAS-37, No. 11, 1990, pp. 1416-1423. doi:10.1109/ 31.62415

[7] C. A. Canizares, N. Mithulananthan, F. Milano and J. Reeve, "Linear Performance Indices to Predict Oscillatory Stability Problems in Power Systems," IEEE Transactions on Power System, Vol. 19, No. 2, 2004, pp. 11041114. doi:10.1109/TPWRS.2003.821460

[8] I. Dobson, J. Zhang, S. Greene, H. Engdahl and P. W. Sauer, "Is Strong Modal Resonance a Precursor to Power System Oscillations?" IEEE Transactions on Circuits and Systems, Vol. 48, No. 3, 2001, pp. 340-349.

[9] V. Vekatasubrumanian, H. Schattler and J. Zaborszky, “A Stability Theory of Large Differential Algebraic Systems: A Taxonomy," Report SSM 9201 — Part I, Washington University, St. Louis, 1992.

[10] V. Vekatasubrumanian, H. Schattler and J. Zaborszky, "Analysis of Local Bifurcation Mechanisms in Large Differential-Algebraic Systems such as the Power System," 
Proceedings of 32nd Conference on Decision and Control, San Antonio, December 1993, pp. 3727-3733.

[11] R. E. Beardmore and R. Laister, "The Flow of a Differential-Algebraic Equation near a Singular Equilibrium," SIAM Journal on Matrix Analysis, Vol. 24, No. 1, 2002, pp. 106-120. doi:10.1137/S0895479800378660

[12] R. E. Beardmore, "The Singularity-Induced Bifurcation and Its Kronecker Normal Form," SIAM Journal on Matrix Analysis, Vol. 23, No. 1, 2001, pp. 1-12.

[13] L. J. Yang and Y. Tang, "An Improved Version of the Singularity Induced Bifurcation Theorem," IEEE Transactions on Automatic Control, Vol. AC-46, No.9, 2001, pp. 1483-1486. doi:10.1109/9.948482

[14] W. Marszalek and S. L. Campbell, "DAEs Arising from Traveling Wave Solutions of PDEs II," Computers and Mathematics with Applications, Vol. 37, 1999, pp. 15-34. doi:10.1016/S0898-1221 (98)00238-7

[15] R. E. Beardmore, "Double Singularity-Induced Bifurcation Points and Singular Hopf Bifurcations," Dynamics and Stability of Systems, Vol. 15, No. 4, 2000, pp. 319-342.

[16] R. Riaza, "On the Singularity-Induced Bifurcation Theorem," IEEE Transactions on Automatic Control, Vol. AC-47, No. 9, 2002, pp. 1520-1523. doi:10.1109/TAC. 2002.802757

[17] S. L. Campbell and W. Marszalek, "Mixed Symbolicnumerical Computations with General DAEs: An Applications Case Study," Numerical Algorithms, Vol. 19, No. 1-4, 1998, pp. 85-94. doi:10.1023/A:1019106507166

[18] S. L. Campbell and W. Marszalek, "DAEs Arising from Traveling Wave Solutions of PDEs I," Journal of Com-

\section{Appendix}

\subsection{Matrix Pencils for First Order Systems}

For a pair of constant square $n \times n$ matrices, say $E$ and $L$, with $\operatorname{det} E=0$, if $\operatorname{det}(s E-L) \neq 0$, then there exist nonsingular matrices $U$ and $V$, such that

$$
U E V=\left(\begin{array}{cc}
I_{m} & 0 \\
0 & N_{n-m}
\end{array}\right), U L V=\left(\begin{array}{cc}
J_{m} & 0 \\
0 & I_{n-m}
\end{array}\right)
$$

where $N_{n-m}$ is a nilpotent matrix of size $(n-m) \times(n-m)$ and index $v \leq n-m$. That is, $v$ is a positive integer such that $N^{v}=0, N^{v-1} \neq 0$. The $I_{k}$ is the unit matrix of size $k \times k$. The pencil ( $U E V, U L V)$ is said to be in Kronecker Normal putational Applied Mathematics, Vol. 82, No. 1-2, 1997, pp. 41-58. doi:10.1016/S0377-0427(97)00084-8

[19] K. L. Praprost and K. A. Loparo, “An Energy Function Method for Determiningvoltage Collapse during a Power System Transient," IEEE Transactions on Circuits System, Vol. CAS-41, No. 11, 1994, pp. 635-651.

[20] M. M. Begovic and A. G. Phadke, "Voltage Stability asSessment of a Reduced State Vector," IEEE Transactions on Circuits System, Vol. 5, No. 1, 1990, pp. 198-203. doi: $10.1109 / 59.49106$

[21] R. Riaza, "Double SIB Points in Differential-Algebraic Systems," IEEE Transactions on Automatic Control, Vol. AC-48, No. 9, 2003, pp. 1625-1629. doi:10.1109/TAC. 2003.817002

[22] Yang Lijun and Zeng Xianwu, "Stability of Singular Hopf Bifurcations," Journal of Differential Equations, Vol. 206, No. 1, 2004, pp. 30-54. doi:10.1016/j.jde.2004. 08.002

[23] R. Riaza, S. L. Campbell and W. Marszalek, "On Singular Equilibria of Index-1 DAEs," Circuits, Systems, and Signal Processing, Vol. 19, No. 2, 2000, pp. 131-157. doi: 10.1007/BF01212467

[24] P. Lancaster and M. Tismenetsky, "The Theory of Matrices," London Academic Press, London, 1985, pp. 454474

[25] J. Guckenheimer, M. Myers and B. Strumfels, "Computing Hopf Bifurcations," SIAM Journal on Numerical Analysis, Vol. 34, No. 1, 1997, pp. 1-2. doi:10.1137/S00 36142993253461

(Canonical) Form and $\sigma(E, L)=\sigma\left(J_{m}\right)$, i.e. the finite spectrum of the pencil $(E, L)$ is the same as the spectrum of matrix $J_{m}$. It is also well-known that for DAEs with the matrix pencil

$$
U E V=\left(\begin{array}{ll}
I & 0 \\
0 & 0
\end{array}\right), U L V=\left(\begin{array}{ll}
A & B \\
C & D
\end{array}\right)
$$

and $\operatorname{det} L \neq 0$, the index $(U E V, U L V)=1$ if and only if $\operatorname{det} D$ $\neq 0$. If $\operatorname{ker}(D)=<v>\neq 0$ and $C B v \notin \operatorname{Im}(D)$, then the index $(U E V, U L V)=2$.

\subsection{Matrix Pencils for Second Order Systems}

The linearization of $\ddot{x}=f(\dot{x}, x, y, \lambda), \varepsilon \dot{y}=g(x, y, \lambda)$ yields the matrix pencil $(E(\varepsilon), L(\lambda))$ with 


$$
E(\varepsilon)=\left(\begin{array}{ccc}
I_{n} & 0 & 0 \\
0 & I_{n} & 0 \\
0 & 0 & \varepsilon I_{m}
\end{array}\right), L(\lambda)=\left(\begin{array}{ccc}
0 & I_{n} & 0 \\
A(\lambda) & \Gamma(\lambda) & B(\lambda) \\
C(\lambda) & 0 & \mathrm{D}(\lambda)
\end{array}\right)
$$

and $A(\lambda)=f_{x}, B(\lambda)=f_{y}, C(\lambda)=g_{x}, D(\lambda)=g_{y}, \Gamma(\lambda)=f_{\dot{x}}$ and $E(\varepsilon), L(\lambda) \in R^{2 n+m}$ and $\operatorname{det}(E(\varepsilon) s-L(\lambda))=\operatorname{det}(E(\varepsilon) s-L(\lambda))$ $=a_{n+m} \mathrm{~s}^{n+m}+a_{n+m-1} s^{n+m-1}+\cdots+a_{1} s+a_{0}$ with $a_{i}=a_{i}(\varepsilon, \lambda)$.

\subsection{Parameter Dependent Polynomials}

The change of the index of DAEs is related to certain properties of the characteristic polynomial of the pencil $(U E V, U L V)$. Let the polynomial be

$$
p(\lambda, s)=a_{r}(\lambda) s^{r}+a_{r-1}(\lambda) s^{r-1}+\cdots+a_{1}(\lambda) s+a_{0}(\lambda)
$$

with $a_{r}\left(\lambda_{0}\right)=a_{r-1}\left(\lambda_{0}\right)=\cdots=a_{r-k+1}\left(\lambda_{0}\right)=0, a_{r-k}\left(\lambda_{0}\right) \neq 0$.

Polynomial (19) admits a real decomposition [13]

$$
p(\lambda, s)=p_{1}(\lambda, s) p_{2}(\lambda, s)
$$

with

$$
\begin{aligned}
& p_{1}(\lambda, s)=c_{k}(\lambda) s^{k}+c_{k-1}(\lambda) s^{k-1}+\cdots+c_{0}(\lambda) \\
& p_{2}(\lambda, s)=s^{r-k}+b_{r-k-1}(\lambda) s^{r-k-1}+\cdots+b_{0}(\lambda)
\end{aligned}
$$

where $c_{k}, \cdots, c_{0}$ and $b_{r-k-1}, \cdots, b_{0}$ are real and smooth, and $c_{k}(\lambda)=a_{r}(\lambda), c_{i}\left(\lambda_{0}\right)=0$ for $1 \leq i \leq k, c_{0}\left(\lambda_{0}\right)=a_{r-k}\left(\lambda_{0}\right) \neq$

$$
R(\lambda)=\left[\begin{array}{cccccccccc}
a_{r-1} & a_{r-3} & a_{r-5} & \cdots & a_{1} & 0 & 0 & 0 & \cdots & 0 \\
0 & a_{r-1} & a_{r-3} & \cdots & a_{3} & a_{1} & 0 & 0 & \cdots & 0 \\
\vdots & & & & & \ddots & & & & \vdots \\
0 & \cdots & 0 & a_{r-1} & a_{r-3} & a_{r-5} & \cdots & a_{3} & a_{1} & 0 \\
a_{r} & a_{r-2} & a_{r-4} & \cdots & a_{2} & a_{0} & 0 & 0 & \cdots & 0 \\
0 & a_{r} & a_{r-2} & \cdots & a_{4} & a_{2} & a_{0} & 0 & \cdots & 0 \\
\vdots & & & & & & & & & \vdots \\
0 & \cdots & 0 & a_{r} & a_{r-2} & a_{r-4} & \cdots & a_{4} & a_{2} & a_{0}
\end{array}\right]
$$

matrix of (19). Note that $\operatorname{det} H_{r}(\lambda)=a_{0} \operatorname{det} H_{r-1}$ and

$$
\operatorname{det} H_{r-1}=(-1)^{r(r+1) / 2} a_{r}^{r-1} \prod_{i, k=1, i<k}^{r}\left(s_{i}+s_{k}\right)
$$

This implies that $\operatorname{det} H_{r-1}$ is zero not only for the purely imaginary roots $\pm j \omega$, but also for a pair of real symmetric roots $s_{1,2}= \pm \sigma$ and for two complex and symmetric pairs of roots $s_{1,2}=\sigma \pm j \omega$ and $s_{3,4}=-\sigma \pm j \omega$. If, during the bifurcation process with $\lambda \rightarrow \lambda_{0}$, a power system operates initially in a stable mode (all eigenvalues are in the open left half plane), then, providing that none of the
0.

If $k=1$, then one root of $p_{1}(\lambda, s)$ diverges through $\infty$ as $\lambda$ $\rightarrow \lambda_{0}$. This is easily seen by observing that with $k=1$ we have $c_{1}(\lambda)=a_{r}(\lambda)=(-1) \operatorname{det} D$, and the root $-c_{0}(\lambda) / c_{1}(\lambda) \rightarrow$ $\infty$ as $\lambda \rightarrow \lambda_{0}$. This divergence is equivalent to the index jump of the matrix pencil from 1 at $\lambda \neq \lambda_{0}$ to 2 at $\lambda=\lambda_{0}$. If $k$ $=2$, then two roots of $p_{1}(\lambda, s)$ diverge through $\infty$ and the index of the corresponding matrix pencil jumps from 1 at $\lambda \neq \lambda_{0}$ to 3 at $\lambda=\lambda_{0}$. The two diverging eigenvalues, say $s_{1,2}$, are such that $\lim _{\lambda \rightarrow \lambda_{0} \mathrm{~s}^{2}}{ }_{1,2}(\lambda)\left(\lambda-\lambda_{0}\right)=\mu$ with $\mu=$ $-\left(u^{T} C\left(\lambda_{0}\right) B\left(\lambda_{0}\right) \mathrm{v}\right) /\left(u^{T} D^{\prime}\left(\lambda_{0}\right) v\right), u \equiv \operatorname{ker} D\left(\lambda_{0}\right)^{T}, v \equiv \operatorname{ker} D\left(\lambda_{0}\right)$, and $D^{\prime} \equiv d D(\lambda) / d \lambda$ (see [1] for more details).

\subsection{Crossing the Imaginary Axis: Loss of Stability}

Applying the Liénard-Chipart splitting [24], polynomial (19) with continuous real coefficients $a_{i}(\lambda)$ can be written as $p(\lambda, s)=p_{\text {even }}\left(\lambda, \mathrm{s}^{2}\right)+s p_{\text {odd }}\left(\lambda, s^{2}\right)$, where

$$
\begin{aligned}
& p_{\text {even }}\left(\lambda,-\omega^{2}\right)=a_{0}(\lambda)+a_{2}(\lambda) s^{2}+a_{4}(\lambda) s^{4}+\left.\cdots\right|_{s=j \omega} \\
& p_{\text {odd }}\left(\lambda,-\omega^{2}\right)=a_{1}(\lambda)+a_{3}(\lambda) s^{2}+a_{5}(\lambda) s^{4}+\left.\cdots\right|_{s=j \omega}
\end{aligned}
$$

Polynomial $p(\lambda, s)$ has purely imaginary roots $\pm j \omega$ if and only if $p_{\text {even }}\left(\lambda,-\omega^{2}\right)=0$ and $p_{\text {odd }}\left(\lambda,-\omega^{2}\right)=0$. These conditions imply that the resultant matrix is singular [25]. Determinant $\operatorname{det} R(\lambda)= \pm \operatorname{det} H_{r}(\lambda)$, where $H_{r}$ is the Hurwitz

eigenvalues diverges through $\infty$, the eigenvalues have to cross the imaginary axis first (at $\lambda_{0}$ ) before reaching unstable locations in either of the symmetric patterns with respect to the origin. Thus, in a typical situation, the increased ill-conditioning of the matrix $H_{r}(\lambda)$ with the changing parameter $\lambda$ will indicate an imminent possibility of the Hopf bifurcation as some of the eigenvalues approach the imaginary axis and become $\pm j \omega$ at $\lambda=\lambda_{0}$ for finite $\omega$. 